\title{
Carl Schmitt, Hermann Heller, Du libéralisme autoritaire
}

\section{Adrien Mathy}

\section{Q OpenEdition}

Journals

Electronic version

URL: http://journals.openedition.org/lectures/45862

ISSN: 2116-5289

Publisher

Centre Max Weber

Brought to you by Université de Liège

\section{LIÈGE \\ université}

Electronic reference

Adrien Mathy, "Carl Schmitt, Hermann Heller, Du libéralisme autoritaire », Lectures [Online], Reviews, Online since 07 December 2020, connection on 07 December 2020. URL : http:// journals.openedition.org/lectures/45862

This text was automatically generated on 7 December 2020

(c) Lectures - Toute reproduction interdite sans autorisation explicite de la rédaction / Any replication is submitted to the authorization of the editors 


\title{
Carl Schmitt, Hermann Heller, Du libéralisme autoritaire
}

\author{
Adrien Mathy
}

1 La notion de néolibéralisme et, avec elle, de libéralisme autoritaire, est au cœur d'enjeux critiques et politiques. Pierre Dardot et Christian Laval soulignaient déjà, en 2009ํํㄹ la nécessité de définir dans toute sa spécificité ce que l'on appelle le néolibéralisme. L'ouvrage élaboré par Grégoire Chamayou, à qui l'on doit déjà une généalogie du libéralisme autoritaire, dans La société ingouvernable (2018) ${ }^{2}$, s'inscrit dans cette optique en apportant un éclairage nouveau sur l'émergence du libéralisme autoritaire. À cet effet, Chamayou confronte deux textes d'auteurs profondément antagonistes : Carl Schmitt (1888-1985) est un conservateur, affilié au parti de centredroit, admirateur de Mussolini, catholique et antisémite, puis collaborateur du régime nazi, tandis qu'Hermann Heller (1891-1833) est un socialiste, membre du SPD, antifasciste, juif et contraint à l'exil. Le premier a été traduit et a joui d'une grande diffusion. Le second n'a malheureusement pas profité d'une pareille popularité. L'ouvrage est ainsi une occasion de nous donner accès à leurs textes. Mais Grégoire Chamayou ne se contente pas de traduire et de présenter ces deux textes. En effet, bien plus que de simples prolégomènes, son « introduction ", qui occupe plus de la moitié de l'ouvrage, propose un travail herméneutique qui permet au lecteur de saisir ce qu'est le libéralisme autoritaire.

Le premier texte est une allocution de Carl Schmitt, prononcée le 23 novembre 1932 devant le patronat allemand. La porte d'entrée que Chamayou utilise pour le présenter est le concept d'État total : «État économique, état fiscal, état d'assistance » (p. 12). De l'État libéral, l'État total est exact opposé ; de plus, il est faible. L'État total est certes total, mais d'un point de vue quantitatif, et non qualitatif. Ainsi, Schmitt diagnostique que le problème de l'Allemagne est cette inflation quantitative de l'État. Pour échapper à cette situation, Schmitt propose, à la suite d'un de ses élèves, Heinz O. Ziegler, non pas de revenir à l'état libéral du XIX ${ }^{\mathrm{e}}$ siècle mais d'en finir avec la démocratie et le parlementarisme, qu'il juge responsables de cette situation. La solution qu'il propose est subsumée par le titre de son allocution : «État fort, économie saine ». À un État total 
quantitatif, Schmitt oppose et propose un État total qualitatif. En effet, pour que l'État se retire de l'économie, pour qu'il la libère, il faut qu'il soit fort. Ainsi, comme le résume Chamayou, «le paradoxe, c'est que, pour avoir moins d'État, il va vous falloir en quelque sorte avoir plus d'État» (p. 19).

Le texte d'Hermann Heller répond à celui de Carl Schmitt. Alors qu'ils entretenaient des liens relativement cordiaux, les deux hommes se sont éloignés à la suite de divergences politiques qui culminèrent en octobre 1932 lors du procès qui fit suite au coup de Prusse, au terme duquel Schmitt identifie définitivement Heller comme un ennemi politique. L'opposition des deux hommes n'est donc pas uniquement interpersonnelle. Heller publie sa réponse à Schmitt en mars 1933 dans la Neue Rundschau. Son article s'intitule "Libéralisme autoritaire? ». Chamayou résume la critique d'Heller en deux points: d'abord, se pose la question des fondations de l'autorité de cet État fort. Chamayou remarque que la conception autocratique de cet État ne s'oppose pas à "l'absence d'autorité, mais bien plutôt au fondement démocratique de celle-ci» (p. 25). Ce constat est d'importance: en effet, l'erreur de la gauche serait de ne pas avoir disputé le terrain du pouvoir au libéralisme autoritaire, et d'être restée cantonnée à une simple défense des droits. Ensuite, se pose la question de l'extension de cette autorité autocratique. Pour Heller, la question consiste à savoir : « envers qui au juste [cet État autoritaire] l'est-il, et avec qui ne l'est-il pas? ? (p. 25).

Une fois les deux textes présentés, l'exposé de Chamayou se concentre principalement sur une analyse de la pensée schmitienne. Il nous semble pouvoir distinguer trois axes dans son interprétation, relatifs aux rapports qu'entretient Schmitt avec le libéralisme, le néolibéralisme et enfin le nazisme. Vis-à-vis du libéralisme, Schmitt peut sembler opposé de prime abord, donc antilibéral : il rejette un certain libéralisme politique, estimant qu'il ne faut pas sacraliser des valeurs issues des Lumières et qui furent certes utiles à l'émancipation de la bourgeoise libérale, mais qui servent à présent «les ennemis de l'ordre» (p.28); il s'oppose par ailleurs à la démocratie libérale, qui ne serait qu'un simulacre de démocratie. Selon lui, il faut une démocratie authentique, une démocratie césariste dont la dictature mussolinienne est le modèle. Enfin, et de façon plus surprenante, Schmitt s'attaque non seulement au contenu politique du libéralisme mais aussi à son contenu économique. Le libéralisme classique, du moins le libéralisme dit manchestérien, partisan du laisser-faire, est selon lui dépassé. Il préconise au contraire que l'État intervienne.

5 À défaut d'être libéral, Schmitt serait-il un néolibéral ? Pour répondre à cette question, Grégoire Chamayou analyse les rapports qu'il entretient avec le nouveau libéralisme naissant. Il constate ainsi que Schmitt est cité avec approbation par Alexander Rüstow et Walter Eucken, précurseurs du néolibéralisme allemand, l'ordolibéralisme. Autrement dit, ils ne le perçoivent nullement comme un antilibéral, bien au contraire. Forts de la théorie schmitienne de l'État total, ces néolibéraux ont expliqué la crise politique de 1929 comme résultant d'une cause externe à l'économie, à savoir une cause politique : l'intrusion étatique, elle-même conséquente à l'intrusion des masses dans la politique. Ainsi, Rüstow attribue le problème à l'État, et « sous l'État, à la démocratie, et sous la démocratie, aux classes laborieuses, aux syndicats, et leurs luttes » (p. 35). Pour Eucken, la solution est simple : il faut que l'État se retire. Les moyens de ce retrait sont abordés dès 1929 par Rüstow dans un texte intitulé «La dictature dans les limites de la démocratie", où il rejoint Schmitt sur la nécessité d'un État fort. Ainsi, le 28 septembre 1932, Rüstow prononce un nouveau discours dont le titre rappelle celui 
de Schmitt : «Économie libre, État fort ». Selon Grégoire Chamayou, le néolibéralisme qui émerge en 1932 en Allemagne est donc interventionniste sur le plan économique et autoritaire sur le pan politique (p. 43). Et il importe de ne pas oublier ce second aspect, trop souvent ignoré par l'historiographie de l'ordolibéralisme.

Enfin, il s'agit d'interroger les rapports entre Schmitt et le nazisme. Cette question est toutefois assez complexe et nécessite d'amener plusieurs éléments contextuels. Il importe de rappeler que si Schmitt est assommé par la nomination d'Hitler à la Chancellerie, c'est parce qu'il était " hors course [...] en tant que conseiller de l'ombre » (p. 53). Son statut de conseiller en fait un des « fossoyeurs de la Weimar» (p. 61), ayant mis dès 1929 ses compétences au service du pouvoir en place, conseillant Papen et avant lui Brüning dont la politique a eu deux conséquences majeures : l'ascension du NSDAP et l'effritement du bloc bourgeois. Dès lors, en l'absence de majorité, la stratégie de Schmitt consiste à «dictatorialiser le pouvoir d'État de l'intérieur, sans prendre appui sur un parti de masse » (p. 65), en usant de l'article 48 pour imposer des mesures économiques. Aussi, à l'automne 1932, Schmitt tente de convaincre le patronat de préférer une voie "moins hasardeuse du point de vue des classes dominantes, à l'aventure hitlérienne»(p.51), à savoir la voie du libéralisme autoritaire. Schmitt prendra ensuite sa carte au parti nazi dès le printemps 1933.

Grégoire Chamayou conclut son analyse sur deux questionnements, le second clôturant son introduction. Premièrement, il s'agit d'interroger la responsabilité du libéralisme autoritaire dans la montée du nazisme. Pour ce faire, il expose et contraste deux thèses : celle de Karl Polanyi qui considère que le libéralisme autoritaire, « loin d'avoir constitué un rempart [...] lui a frayé un chemin» (p. 73); et celle de Friedrich Hayek, disciple de Schmitt qui, au contraire, estime que le nazisme est le résultat nécessaire des tendances socialistes, estimant, in fine, que le responsable est la démocratie, en tant qu'État total quantitatif. Pour Chamayou, Hayek réalise un tour de force révisionniste et transpose finalement le discours schmittien aux démocraties occidentales d'aprèsguerre. Deuxièmement, Grégoire Chamayou interroge la lecture foucaldienne du néolibéralisme. Il remarque que si Foucault a bien identifié l'interventionnisme libéral propre au néolibéralisme, il a négligé l'autoritarisme libéral. Foucault commence sa généalogie du néolibéralisme et de l'ordolibéralisme en 1948, considérant ce dernier comme une réaction au nazisme. Cependant, à cette lecture, Chamayou en oppose une autre, sur la base de son analyse des textes de Schmitt et d'Heller. Le néolibéralisme, et sa facette autoritaire, émergent contre la démocratie libérale. Fort de cette conclusion, Chamayou mobilise le travail critique que représente cet ouvrage afin de penser la crise de gouvernementalité du néolibéralisme actuel.

8 À la lecture de l'ouvrage, il est difficile de ne pas dresser des parallèles avec le monde contemporain. Grégoire Chamayou assume d'ailleurs cette comparaison en conclusion de son travail. Par ailleurs, l'attention proportionnellement moindre apportée au texte d'Heller donne au lecteur la sensation que l'intérêt de l'ouvrage est surtout dans la critique des travaux de Schmitt. Cette critique poursuit certes une approche généalogique mais, en tant que telle, elle participe aussi d'une logique, sinon militante, de lutte contre le libéralisme autoritaire et le néolibéralisme - ce qui a pu être reproché à son précédent ouvrage ${ }^{3}$. Enfin, Grégoire Chamayou conclut sur le constat d'un virage autoritaire en France, auquel il répond en citant Herman Heller : " "Autoritaire" et fort, un tel État se doit de l'être, [...]. Car, dans des formes démocratiques, le peuple allemand ne tolèrerait pas longtemps cet État néolibéral » (p. 82). 


\section{NOTES}

1. Pierre Dardot, Christian Laval, La nouvelle raison du monde. Essai sur la société néolibérale, Paris, La Découverte, 2009 ; compte rendu de Jean-Luc Metzger pour Lectures : https://doi.org/10.4000/ lectures.910.

2. Grégoire Chamayou, La société ingouvernable. Une généalogie du libéralisme autoritaire, Paris, La Fabrique, 2018; note critique de Thibault Le Texier pour Lectures: https://doi.org/10.4000/ lectures.34765.

3. Ibid.

\section{AUTHOR}

\section{ADRIEN MATHY}

Responsable scientifique (ULiège Library/Université de Liège). Doctorant au sein du Centre de sémiotique \& rhétorique (Université de Liège). 